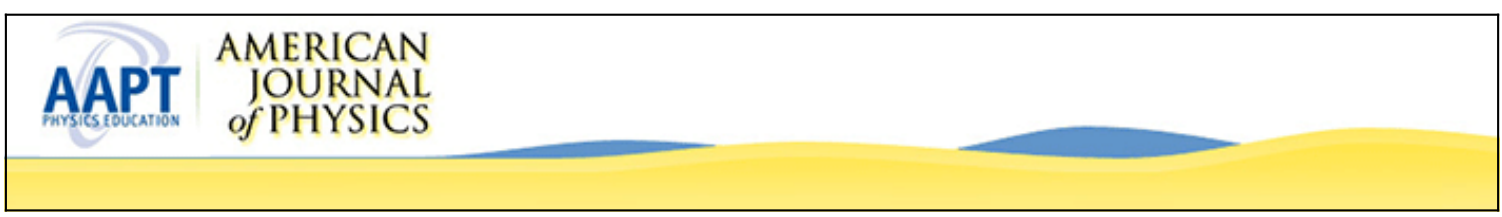

\title{
Microwave attenuation in liquid media
}

Yiannis H. Haranas and W. P. Lonc

Citation: American Journal of Physics 52, 214 (1984); doi: 10.1119/1.13694

View online: http://dx.doi.org/10.1119/1.13694

View Table of Contents: http://scitation.aip.org/content/aapt/journal/ajp/52/3?ver=pdfcov

Published by the American Association of Physics Teachers

\section{Articles you may be interested in}

SHOCK ATTENUATION IN DISTENDED MEDIA

AIP Conf. Proc. 1195, 1031 (2009); 10.1063/1.3294975

Broadband microwave absorption spectrometer for liquid media

Rev. Sci. Instrum. 59, 2577 (1988); 10.1063/1.1139901

Absorption and attenuation in biological media

J. Acoust. Soc. Am. 84, S137 (1988); 10.1121/1.2025796

Ultrasound fields in attenuating media

J. Acoust. Soc. Am. 80, 1140 (1986); 10.1121/1.393804

Covariance of Noise in Attenuating Media

J. Acoust. Soc. Am. 36, 1183 (1964); 10.1121/1.1919181

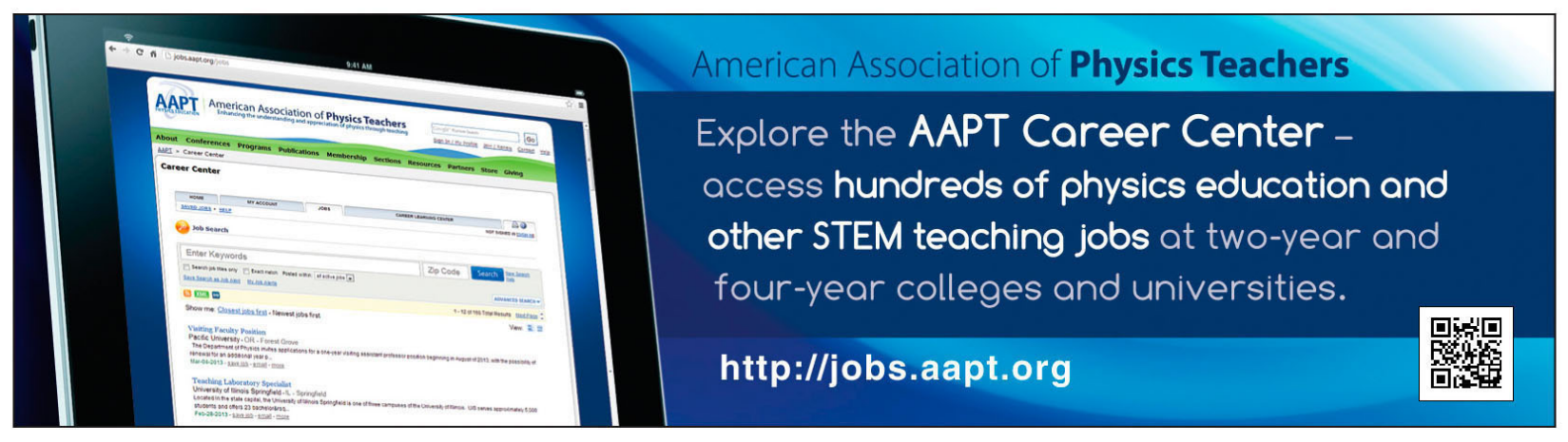


${ }^{12}$ E. E. Salpeter, in Perspectives in Modern Physics, edited by R. E. Marshak (Wiley, New York, 1966), pp. 463-475.

${ }^{13}$ V. F. Weisskopf, Science 187, 605-612 (1975).

${ }^{14}$ C. W. Allen, Astrophysical Quantities (Athlone, London, 1976).
${ }^{15}$ G. Wald, Sci. Am. 201, 92-108 (1959)

${ }^{16} \mathrm{~B}$. M. Oliver, in The Search for Extraterrestrial Intelligence, NASA SP. 419, edited by P. Morrison, J. Billingham, and J. Wolfe (NASA, Washington, DC, 1977).

\title{
Microwave attenuation in liquid media
}

\author{
Yiannis $\mathrm{H}$. Haranas and W. P. Lonc, S. J. \\ Physics Department, Saint Mary's University, Halifax, Nova Scotia B3H 3C3, Canada
}

(Received 18 January 1982; accepted for publication 3 June 1983)

A semi-quantitative undergraduate experiment is described which verifies that the attenuation of microwaves propagating in a lossy liquid medium depends both on the distance traveled by the wave in the medium, as well as on the medium's electric conductivity.

\section{INTRODUCTION}

An initial study, at the undergraduate level, of the relationship between the intensity of electromagnetic radiation traveled and the conductivity of the medium may be carried out at microwave frequencies in the $10-\mathrm{GHz}$ range, for example, using rather simple equipment. The objective of this paper is to make available to the readers of this Journal a tested experiment which could be used as a laboratory project or exercise. The experiment should take no more than three hours, and verifies the following: first, that for a given lossy liquid medium (ordinary tap water with some common salt added), the intensity of the propagating wave drops off exponentially with distance traveled in the medium, and second, that for a given distance traveled in such a medium the intensity of the propagating wave is a decreasing function of the medium's electric conductivity.

In this discussion, it is assumed that the conductivity $g$ is a macroscopic quantity representing the overall contributions from various microscopic mechanisms, such as molecular rotation, scattering, etc. ${ }^{1}$ Further, it is being assumed that the macroscopic conductivity in this experiment varies only with the salt concentration.

In other words, in the first case, the experiment verifies the exponential form of the space-dependent factor describing the wave in a lossy medium (tap water), rather than the actual value of the medium's attenuation properties. In the second case, the experiment verifies that a given liquid medium - an aqueous solution of common table salt in tap water-will exhibit an attenuation which increases as the conductivity increases. The form of this relationship is not investigated in the experiment.

In both cases, questions concerning the chemical properties of the liquid media are circumvented by simply using the available drinking water as the medium with the initially lowest conductivity (and hence, the lowest loss), and then adding some ordinary table salt to provide a medium with a definitely higher (albeit unknown) electric conductivity. The saline solution is expected to exhibit a larger loss or attenuation of the microwaves propagating through this medium.

\section{THEORY}

From standard treatments of electromagnetic theory at the undergraduate level, ${ }^{2}$ it is shown that the intensity of plane waves propagating along the $z$ axis in a medium described by an attentuation factor $\alpha$ may be expressed as

$$
I(g, z) \propto e^{-\alpha z},
$$

where $I(g, z)$ is the measured intensity of the wave, and where $\alpha$ is a function of $g$. Figure 1 gives a representation of a plane wave propagating through a lossy medium. Within the lossy medium, the wave amplitude is attenuated in an exponential manner. For various (say, two) values of the conductivity $g$, a semilogarithmic plot of the intensity as a function of $z$ would yield straight lines, as shown in Fig. 2.

\section{EXPERIMENTAL PROCEDURE}

The basic apparatus is shown in Fig. 3, in which 10-GHz electromagnetic radiation is arranged to propagate through a layer of liquid. The thickness of the layer is variable, so that the receiver, which is at a fixed location during the experiment, primarily measures the "free-space" intensity of the wave which has propagated through a layer of lossy medium a distance $z=b$.

It is assumed that the radiation intensity at any point along the propagation path consists of a number of compo-

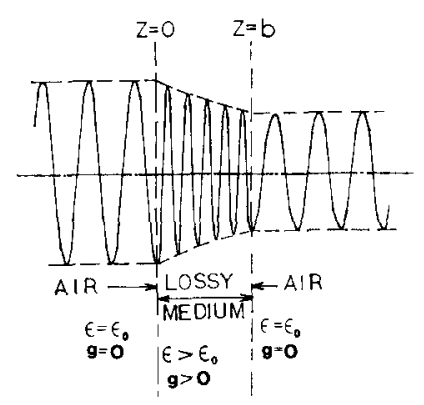

Fig. 1. Simplified representation of a traveling wave at some arbitrary instant of time, showing transmission through a region of lossy medium. Note that the apparent wavelength of the radiation is reduced as it is propagating through the medium in which the permittivity is larger than for free space. Note also that the amplitude of the propagating wave decreases exponentially in the lossy medium. 


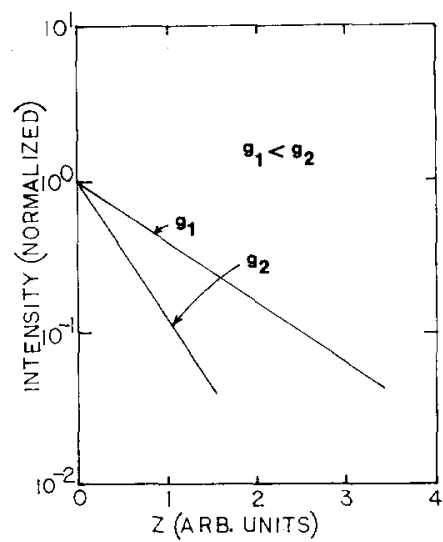

Fig. 2. Representation of the expected behavior of the observed intensity $I_{\exp }(g, z)$, for two arbitrary values of conductivity, $g_{1}$ and $g_{2}$, where $g_{1}<g_{2}$.

nents, some of which represent reflections from various surfaces in the path of propagation. And, since the electric conductivity of the untreated water is already relatively large, then those components associated with reflection within the liquid medium should be relatively small by the time they reach the receiver.

As for other reflected components, such as the one associated with reflection from the receiver itself back to the surface at $z=b$, it is argued that here again the intensity is relatively small, partly because the radiation is not significantly collimated, and partly because some of it re enters the liquid where it is attenuated even further.

In general, given that the experiment is meant to illustrate absorption effects in a semi quantitative manner rather than to actually determine the absorption coefficient for a particular medium, then the argument given above pertaining to negligible effects is fairly plausible. In fact, by way of post factum proof, the results of the experiment as shown in Fig. 4 do, indeed, give support to the claim that the only significant radiation reaching the receiver is that associated with the direct wave and described by the expression in Eq. (1).

As indicated in Fig. 3, the microwave radiation enters the liquid at the bottom of the tank, and is partially collimated by the transmitter horn. The transmitter horn serves to increase somewhat the amount of available power launched into the liquid medium. The microwaves then travel some distance, from $z=0$ to $z=b$ (where $b$ is variable) within the liquid, undergoing attenuation. At $z=b$, the waves are then traveling in air again, for which the electric conductivity is practically zero-hence, negligible attenuation-and then reach the receiver horn. The receiver radiation subsequently produces a deflection on the meter $M$. The two metal sheets $S_{1}$ and $S_{2}$ serve to shield the

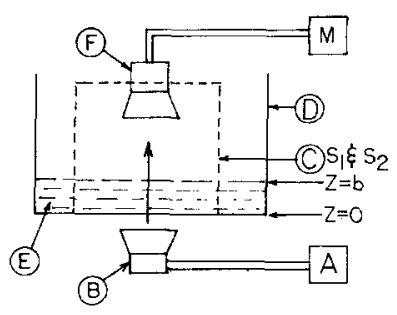

Fig. 3. Block diagram of the experimental apparatus (not to scale). A: microwave source (including variable attenuator), feeding the transmitting horn; B: transmitting horn; C: outline of metal shields $S_{1}$ and $S_{2}$, straddling the receiving horn; $D$ : liquid container (small aquarium, $35 \times 12 \times 20 \mathrm{~cm}$ ); E: liquid medium; F: receiving horn; and $M$ : voltmeter.

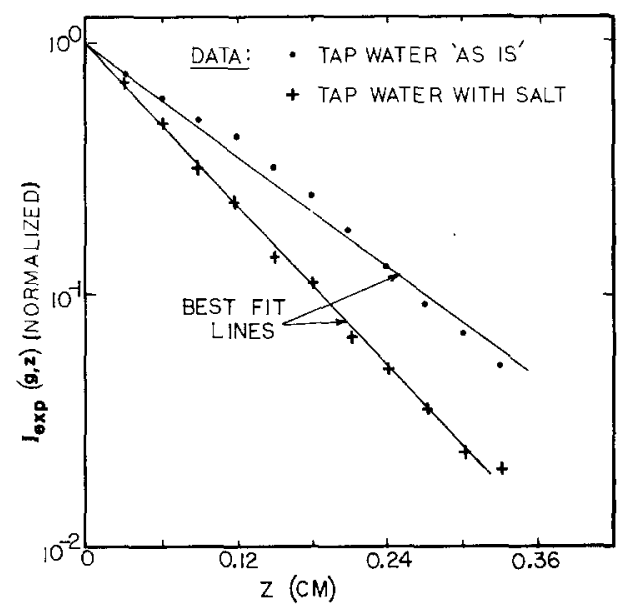

Fig. 4. Experimental results, showing the normalized observed intensity as a function of two different conductivities: one, for tap water "as is," and the other for the same water with some table salt added. The data points represent the average of several runs.

detector from any radiation that may propagate along the external surface of the tank.

It is assumed that the detection element is a "squarelaw" device, so that the meter reading has a fixed relationship to the received power. If the detection element is a semi conducting diode, then this condition is usually satisfied sufficiently well if the radiation source has a power output in the order of a few milliwatts. This is the case for the usual microwave sources such as klystrons (local oscillator types), Gunn diodes, etc. Operation in the the "square-law" region may be presumed to exist if the meter produces a deflection which is associated with a crystal current of no more than, say, a few microamperes. This would correspond to a few millivolts on a voltmeter with an input resistance in the order of $10 \mathrm{k} \Omega$.

In the actual performance of the experiment, enough liquid is poured into the tank to cover the bottom of the tank. Any irregularities existing on the bottom surface are expected to be ineffectual if the thickness of the liquid medium is great enough. In our experiment, the starting thickness was in the order of $0.2 \mathrm{~cm}$. With this amount of liquid in the tank, and with the meter set at a sensitivity consistent with "square-law" behavior in the detecting element, the waveguide attenuator is adjusted to produce a full-scale deflection (or some other convenient value). The top surface of the liquid is now taken to be the plane $z=0$. Hence, the deflection on the meter and $z=0$ define the first data point to be plotted on a semi logarithmic graph. A measured amount of liquid is now added to that already in the tank so that the corresponding increase in the length of the liquid medium is known - this increase in thickness is simply the next value of $z$-and the associated meter deflection is noted. These two quantities define the second data point, and so on.

To decide on the amount by which the length of the liquid medium should be increased for successive data points, consideration could be given to the fact that the apparent wavelength decreases with increasing permittivity, it being assumed that the permeability is essentially that of free space. ${ }^{3}$ If there is, in fact, a significant standing wave in the liquid medium for some values of $z$, then the standing-wave pattern would be detected if the data points are 
obtained at multiples of a small fraction of the wavelength in that medium.

Assuming that the dielectric constant of the untreated water is nominally equal to 60 , and that this value does not change significantly for small amounts of added salt, then the wavelength of $10 \mathrm{GHz}$ waves propagating though such a medium would be approximately $0.4 \mathrm{~cm}$, so that increases in the path length within the liquid in steps of perhaps 0.04 $\mathrm{cm}$ should suffice to detect any existing standing-wave pattern. Inspection of the data for the lower conductivity in Fig. 4 does suggest the presence of a standing wave, but its amplitude is seen to be relatively small compared with the dominant trend of the data.

As mentioned above, the starting medium was chosen to be locally available drinking water "as is." A nonrigorous test or measure of the conductivity of this water may be made by simply immersing both leads of an ohmmeter into the water. In our experiment, using a battery-operated volt-ohm meter (VOM) on the RX1K scale, with the test leads $1 \mathrm{~cm}$ apart, the semi quantitative measurement indicated a resistance of approximately $20 \mathrm{k} \Omega$. After the salt was added $(20 \mathrm{~g}$ per $500 \mathrm{mil}$ of water), the resistance was approximately $4 \mathrm{k} \Omega$.

\section{EXPERIMENTAL RESULTS}

Inspection of Fig. 4 indicates that the data points do tend to define a straight line on the semilogarithmic coordinates, indicating that the behavior may be described mathematically by an exponential function with a negative exponent. Moreover, the data indicate that the attenuation is more rapid for the medium with the larger electric conductivity. Inspection of the data for the case of lower conductivity indicates a fairly clear sinusoidal scatter of the data points, suggesting that a noticeable standing wave pattern exists in the medium, and the apparent wavelength appears to be approximately the expected value. Moreover, inspection of the data for the larger conductivity indicates no standing wave pattern, which agrees with the expectation that standing wave patterns in media with higher conductivities will be attenuated progressively more rapidly.

\section{CONCLUSION}

In reference to the objective in this experiment, which was to verify that in a given lossy medium, the intensity of the propagating electromagnetic wave drops off exponentially with distance traveled in the medium, and that for a given distance traveled the intensity of the wave is a decreasing function of the medium's electric conductivity, it is concluded that the experiment described in this paper does, indeed, achieve this objective.

\footnotetext{
'Further information on the microwave dielectric properties of water is found, for example, in the article by C. W. Kern and M. Karplus, in The Physics and Physical Chemistry of Water, edited by Felix Franks (Plenum, New York, 1972), pp. 276ff; J. B. Hastead, Aqueous Dielectrics (Chapman and Hall, London, 1973), pp. 43ff. For the purpose of this paper, the microscopic basis for the conductivity of water is not at issue. ${ }^{2}$ J. D. Jackson, Classical Electrodynamics (Wiley, New York, 1962), p. 224; P. Lorrain and D. R. Corson, Electromagnetic Fields and Waves (Freeman, San Francisco, 1970), Chap. 11; David M. Cook, The Theory of the Electromagnetic Field (Prentice Hall, Englewood Cliffs, NJ, 1975), Chap. 13; Arthur F. Kip, Fundamentals of Electricity and Magnetism (McGraw-Hill, New York, 1969), Appendix L. Note that our paper follows the treatments presented in the references above inasmuch as the "conductivity" $g$ is being taken in a phenomenological or macroscopic sense.

${ }^{3}$ The static dielectric constant of water at room temperature is given in the literature as approximately 78 ; this is sometimes called the "low-frequency" value. This value is given, for example, in Handbook of Chemistry and Physics (CRC, Boca Raton, FL, 1981), 62nd ed., p. E-58.
}

\title{
Measurement of fluid flow using streak photography
}

\author{
T. D. Dickey, B. Hartman, E. Hurst, and S. Isenogle \\ Department of Geological Sciences, University of Southern California, Los Angeles, California 90089-0741
}

(Received 7 February 1983; accepted for publication 8 June 1983)

Streak photography has been used primarily for fluid flow visualizations. A method which extends the application of streak photography to velocity measurements is presented. The technique may be applied to a broad range of fluid dynamical experiments and requires only limited technical resources.

\section{INTRODUCTION}

Fluid flow problems are currently being studied in the laboratory with a variety of experimental techniques. ${ }^{1.2}$ Hot-wire and hot-film anemometry are used primarily in air and water studies, respectively. Both techniques are based upon the principle that changes in fluid velocity can be detected by heated conductivity probes. These methods enable high-frequency $(\sim 50000-\mathrm{Hz})$ measurements and are ideal for time series analysis. However, relatively com- plex and expensive data acquisition systems must be employed. In addition, the influence of a probe upon the flow field must be considered. Acoustic and laser anemometry techniques utilize the Doppler effect and enable measurements free of probe disturbances. Unfortunately, these methods are also technically demanding and often cost prohibitive.

Streak photography of a variety of tracer materials (e.g., aluminum flakes, oils, etc.) has been used for qualitative flow visualizations. ${ }^{1}$ One major advantage of this method is 